\title{
TIPOLOGÍA DE FINCAS CACAOTERAS EN LA SUBCUENCA MEDIA DEL RÍO HUAYABAMBA, DISTRITO DE HUICUNGO (SAN MARTÍN, PERÚ)
}

\section{COCOA FARM TYPOLOGY IN THE MID SUB-BASIN OF HUAYABAMBA RIVER IN THE HUICUNGO DISTRICT- SAN MARTÍN- PERU}

\author{
Oscar Tuesta Hidalgo ${ }^{1}$, Alberto Julca Otiniano², Ricardo Borjas Ventura ${ }^{2}$, Patricia Rodríguez Quispe ${ }^{2}$ y \\ Mercedes Santistevan Méndez ${ }^{3}$
}

\begin{abstract}
Resumen
Este trabajo se realizó con el objetivo de tipificar las fincas cacaoteras en la subcuenca media del río Huayabamba, distrito de Huicungo (San Martín, Perú). Se trabajó con la Cooperativa Agraria Cacaotera ACOPAGRO con una población de 332 productores y de la que se tomó una muestra (n=77) para hacer una encuesta que consideró 6 componentes (familiar, recursos naturales, actividad agrícola, económico, tecnológico y de gestión, social). Se encontró que las fincas cacaoteras están agrupadas en tres tipos, significativamente diferenciados entre sí. El grupo I fue el mayoritario (68\% de las fincas), se caracteriza por desarrollar otras actividades paralelas al cultivo del cacao, tiene un sistema de producción convencional, con plantaciones policlonales y un rendimiento promedio de $963.46 \mathrm{~kg} . \mathrm{ha}^{-1}$, cifra que supera la media regional y nacional. El grupo II (13 \% de fincas) reúne básicamente fincas de producción orgánica, donde predominan plantaciones hibridas asociadas con CCN51 que tienen un rendimiento que no superan el rendimiento regional; pero si el nacional. En el grupo III, se encuentran plantaciones monoclonales usando únicamente el CCN-51. Los resultados sugieren que si en el futuro se implementan proyectos de mejora tecnológica u otros, estos deberían ser específicos para cada uno de los tres grupos encontrados en esta zona de estudio.
\end{abstract}

Palabras clave: Agroforestería, cacao, tipificación, San Martín, Perú.

\begin{abstract}
This research was carried out in order to typify cocoa farms located in the middle Huayabamba River basin, Huicungo district, San Martín, Peru. We worked with the ACOPAGRO Cocoa Agricultural Cooperative from which a sample $(n=77)$ was taken from a population of 332 farmers. A survey was conducted that took into account six components (family, natural resources, agricultural activity, economics, technology and social management). It was found out that cocoa farms are grouped into three types, significantly differentiated from each other. Group I was the major group (68\% of the farmers). Farmers in this group also perform other farming activities besides cocoa farming; they practice a conventional production system based on polyclonal plantations and have an average yield of $963.46 \mathrm{~kg} \mathrm{ha}^{-1}$, which is higher than the regional and national average. Group II (13\% of the farms) basically includes organic production farms having hybrid plantations associated with CCN51, with yields lower than the regional average but higher than the national average. Group III, includes monoclonal plantations planted only with CCN-51. The results suggest that if future projects of technological improvements or others are implemented, they should be specific for each one of the three groups found in this study area.
\end{abstract}

Key words: Agroforestry, cocoa, typology, San Martin, Peru.

\section{Introducción.}

En una región es posible hallar factores físicos, biológicos, socioeconómicos y culturales diversas asociadas con las unidades de producción agropecuaria, por eso es conveniente identificar las distintas combinaciones de esos factores y su relación con los diversos tipos de unidades de producción presentes en un área geográfica determinada (Rodríguez, 2002). Las técnicas convencionales utilizadas para identificar grupos objetivos de proyectos de investigación y transferencia de tecnología agropecuaria, generalmente no han logrado dar cuenta de la diversidad de fincas existentes en la mayoría de regiones en que el problema ha sido analizado críticamente (Escobar \& Berdegué, 1990). Quizá, por ello los proyectos de investigación se han centrado en el desarrollo de marcos teóricos y en la aplicación de técnicas de clasificación de fincas, que 
faciliten agrupar aquellas con condiciones homogéneas y permitan determinar, en forma clara, grupos de trabajo caracterizados, en los cuales sea posible conocer limitaciones y posibilidades para la introducción de cambios tecnológicos (Escobar, 1986). Y es que, las experiencias indican, que la selección de áreas para desarrollar proyectos de generación de tecnologías tiene usualmente un alto grado de heterogeneidad. Esto como resultado no solamente de las condiciones ecológicas, sino también de aspectos económicos de la estructura de producción, la disponibilidad de factores de producción y la influencia de condiciones macro, como la especialización del mercado y el acceso a los servicios y la infraestructura necesaria para la producción (Escobar, 1986). La tipificación, entre otras cosas, permite conocer la organización conceptual de la diversidad existente en la agricultura campesina; una lista de unidades de producción representativa y la población vinculada a los sistemas de producción (Escobar \& Berdegué 1990).

Por otra parte, el cultivo de cacao en el Perú ha despertado gran interés, debido al alza de precios y a la demanda aún insatisfecha tanto del mercado nacional e internacional; pero de manera especial este último. En el 2013 nuestro país exportó $60 \mathrm{mil}$ toneladas de cacao que representó US\$ 116.6 millones y generaron alrededor de 6.3 millones de jornales, beneficiando directamente a $40 \mathrm{mil}$ familias e indirectamente a 200 mil personas. Según el MINAG (2005), el rendimiento promedio nacional (513 kg/ha), es superior al promedio mundial, estimado en 470 $\mathrm{kg} / \mathrm{ha}$. Pero existen reportes de rendimientos mayores al promedio nacional en diversas localidades productoras de cacao. San Martín es una importante región productora de cacao en la zona nor-oriental del país, ahí las fincas de la subcuenca media del río Huayabamba, presentan distintas características productivas y socioeconómicas que impactan de forma diferente sobre el ecosistema. Para el diseño de programas de desarrollo, es importante hacer una clasificación de las fincas de esta subcuenca buscando que no impacten negativamente sobre el ecosistema. Por ello, este trabajo tuvo como objetivo tipificar las fincas cacaoteras en la subcuenca media del río Huayabamba, distrito de Huicungo en la región San Martín.

\section{Materiales y métodos.}

El estudio se realizó en la parte media de la subcuenca del río Huayabamba, localizada en la región San Martín provincia de Mariscal Cáceres el distrito de Huicungo (Figura 1). El territorio donde está asentada la población beneficiaria directa se extiende en un ámbito de 20754.49 ha.

De acuerdo al mapa ecológico del Perú, la zona se ha identificado como un bosque húmedo tropical (bht). El promedio de precipitación anual es de 1200

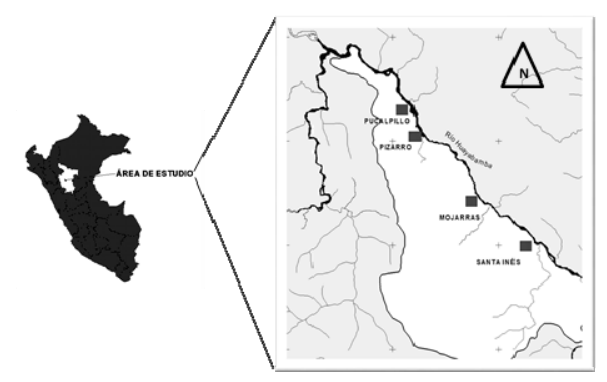

Figura 1. Área de estudio, Subcuenca del río Huayabamba. Fuente: Rodríguez (2005).

$\mathrm{mm}$, la temperatura media anual es de $30^{\circ} \mathrm{C}$, con una media anual máxima de $35^{\circ} \mathrm{C}$ y la media anual mínima de $23.2^{\circ} \mathrm{C}$. Se trabajó con la Cooperativa Agraria Cacaotera ACOPAGRO, la que tiene una población de 332 productores y se tomó una muestra de 77 productores. Para determinar el tamaño de la muestra $(\mathrm{n}=77)$, se usó a la fórmula de Scheaffer et al. (1987). La colecta de datos se realizó a través de una encuesta que consideró seis componentes (Familiar, Recursos Naturales, Actividad Agrícola, Económico, Tecnológico y de Gestión, Social) teniendo un total de 30 variables (18 cuantitativas y 12 cualitativas), tal como se muestra en la Tabla 1.

La encuesta fue procesada en una hoja de cálculo, para determinar el agrupamiento de fincas se realizó un análisis de conglomerados (cluster analysis), por el método de Ward (Andrade, 1985). El método de Ward conforma grupos utilizando el Análisis de Varianza (ANDEVA), donde la variabilidad dentro de grupos es mínima y entre grupos es máxima. Asimismo se realizó el Análisis de varianza multivariada para las variables cuantitativas y así poder determinar diferencias significativas entre los grupos encontrados, y la Prueba de Duncan para comprobar la significancia de cada variable seleccionada dentro del conglomerado. Para las variables cualitativas se realizaron tablas de contingencia utilizando Chi cuadrado para definir diferencias estadísticas entre grupos. También se empleó estadística descriptiva para describir ciertas características de los grupos formados. El análisis de conglomerados se realizó utilizando las 30 variables de la encuesta realizada. Las variables cualitativas se convirtieron a numéricas dicotómicas $(0,1)$ para facilitar el procesamiento, los grupos identificados fueron caracterizados y diferenciados entre sí, mostrando la potencialidad así como los limitantes de cada grupo identificado. Se realizó el Análisis de Componentes Principales para identificar las variables que influyen sobre la conformación de grupos formados; asimismo, permitió identificar variables altamente correlacionadas entre ellas. El análisis discriminante canónico fue realizado tomando en cuenta la agrupación generada por el Análisis de Conglomerados, esto permitió reducir la dimensión de 
Tabla1. Componentes en estudio y sus respectivas variables.

\begin{tabular}{|c|c|}
\hline \multicolumn{2}{|c|}{ VARIABLES EN ESTUDIO } \\
\hline FAMILIAR & $\begin{array}{l}\text { TECNOLOGÍA Y } \\
\text { GESTIÓN }\end{array}$ \\
\hline Edad del productor (años) & $\begin{array}{l}\text { Área de } \\
\text { construcciones }\left(\mathrm{m}^{2}\right)\end{array}$ \\
\hline Número personas por familia & Genotipos сасао \\
\hline Originario de la localidad & Realiza poda \\
\hline RECURSOS NATURALES & $\begin{array}{l}\text { Número de } \\
\text { deshierbos al año }\end{array}$ \\
\hline Ojo de agua en la finca & $\begin{array}{l}\text { Control de plagas } \\
\text { cultural }\end{array}$ \\
\hline Río o quebrada en la finca & $\begin{array}{l}\text { Venta a la } \\
\text { Cooperativa }\end{array}$ \\
\hline Uso de agua de pozo & Lleva registros \\
\hline Contaminación del agua & SOCIAL \\
\hline Erosión en la finca & $\begin{array}{l}\text { Aconsejado por } \\
\text { extensionista }\end{array}$ \\
\hline $\begin{array}{l}\text { Número de especies forestales en } \\
\text { parcela de cacao }\end{array}$ & $\begin{array}{l}\text { Pertenece a } \\
\text { organización }\end{array}$ \\
\hline Área de bosque (ha) & $\begin{array}{l}\text { Tiempo organizado } \\
\text { (años) }\end{array}$ \\
\hline Área de purma (ha) & \\
\hline ACTIVIDAD AGRÍCOLA & \\
\hline Principales actividades & \\
\hline Área con cultivo de cacao (ha) & \\
\hline Edad del cultivo de cacao (años) & \\
\hline Área total del fundo (ha) & \\
\hline Área de cultivos anuales (ha) & \\
\hline ECONÓMICO & \\
\hline Producción anual de cacao (kg/ha) & \\
\hline Mano de obra (escala) & \\
\hline Margen Bruto (S/.x ha)* & \\
\hline Financiamiento externo & \\
\hline
\end{tabular}

(*) Resultado de la encuesta, considera los ingresos por venta de la producción de grano y los costos de producción,

las variables, excluyendo las variables de bajo poder discriminante.

\section{Resultados y discusión.}

Como se puede observar en la Figura 2, se definieron tres grupos de fincas, el primer grupo está formado por 52 fincas (68\%), mientras que el segundo grupo está formado por 10 fincas (13\%) y por último el tercer grupo conformado por 15 fincas (19\%). Resultados similares se ha reportado en otros países productores de cacao, donde este cultivo, también es parte de diversos sistemas agroforestales que se pueden agrupar en función de las características más importantes que tenga un grupo comparado con otro (Nair, 1982; Altieri, 2002, Salazar, 2012).

Las variables del componente Familiar no resultaron significativas (Tabla 2), sin embargo es necesario observar que el número de personas por familia va de tres a cuatro. Respecto a si los agricultores son originarios de la localidad, en las fincas del grupo II, la mitad de ellas vienen de otras localidades, mientras que en el grupo I y III, la mayoría de agricultores son de la localidad (Figura 3).
Esta información es importante, ya que los proyectos que intervienen en un área geográfica determinada, deben considerar con qué tipo de beneficiarios van a trabajar, se debe respetar mucho las costumbres y tradiciones, para evitar conflictos entre la organización ejecutora y la población.

En relación a las variables del componente Recursos Naturales, observamos que el número de especies forestales presentó diferencias significativas entre grupos (Tabla 2), los grupos I y III, tienen en sus plantaciones de cacao, otras especies arbóreas y en mayor número que los del grupo II. Esto es importante porque el uso de plantaciones agroforestales en cacao, permite conciliar objetivos múltiples de producción y conservación a largo plazo, contribuye a controlar la erosión de los suelos ubicados en zonas de alta pendiente, logrando mantener la fertilidad del suelo, ayuda a la sostenibilidad ambiental y el uso adecuado de los recursos naturales (Jiménez, 2002).

Las fincas de los grupos en estudio están ubicadas en suelos no aptos para el desarrollo de actividad agrícola, por la condición de pendiente y de la cercanía a la zona de amortiguamiento del Parque Nacional Río Abiseo. Es probable, que los únicos sistemas que pueden disminuir el impacto negativo sobre dicho parque, son los sistemas agroforestales por las prácticas conservacionistas del suelo (Somarriba, 2006). Una posible ventaja de estos sistemas es que los arboles pueden plantarse en campos agrícolas en múltiples formas (Raintree, 1987) y ajustados a las condiciones de las fincas, de los sistemas de producción y objetivos de los productores (Somarriba, 1998). Además del efecto sobre el medio ambiente, esta técnica puede aportar mayor ingreso a los agricultores de la zona, ya que se ha reportado que en el Alto Beni (Bolivia), los agricultores que realizan agroforesteria podrían tener arboles con un equivalente a 2 ha de reforestación por finca (Vega \& Somarriba, 2005). No solo hay un aumento de ingresos para el agricultor; sino también una disminución de costos cuando existen cultivos asociados (Corven, 1993).

Por otro lado, las fincas del grupo I y III, son las que presentan algún grado de erosión en comparación del grupo II que no muestra signos de erosión. Esto a pesar que el área con bosques es mayor en el grupo I, en comparación con II y III, diferencia que no es estadísticamente significativa (Tabla 2). Estos resultados, sugieren que no solo se debe evaluar el tamaño del área con bosques; sino también otros aspectos del bosque, como la composición botánica, edad de los árboles, etc. Caso contrario podríamos tener una interpretación inadecuada del papel que tendría el bosque para disminuir el riesgo de erosión. Esto es importante si consideramos que entre las características de los suelos erosionados está la pérdida de materia orgánica y la disminución permanente de la fertilidad, efecto que puede 


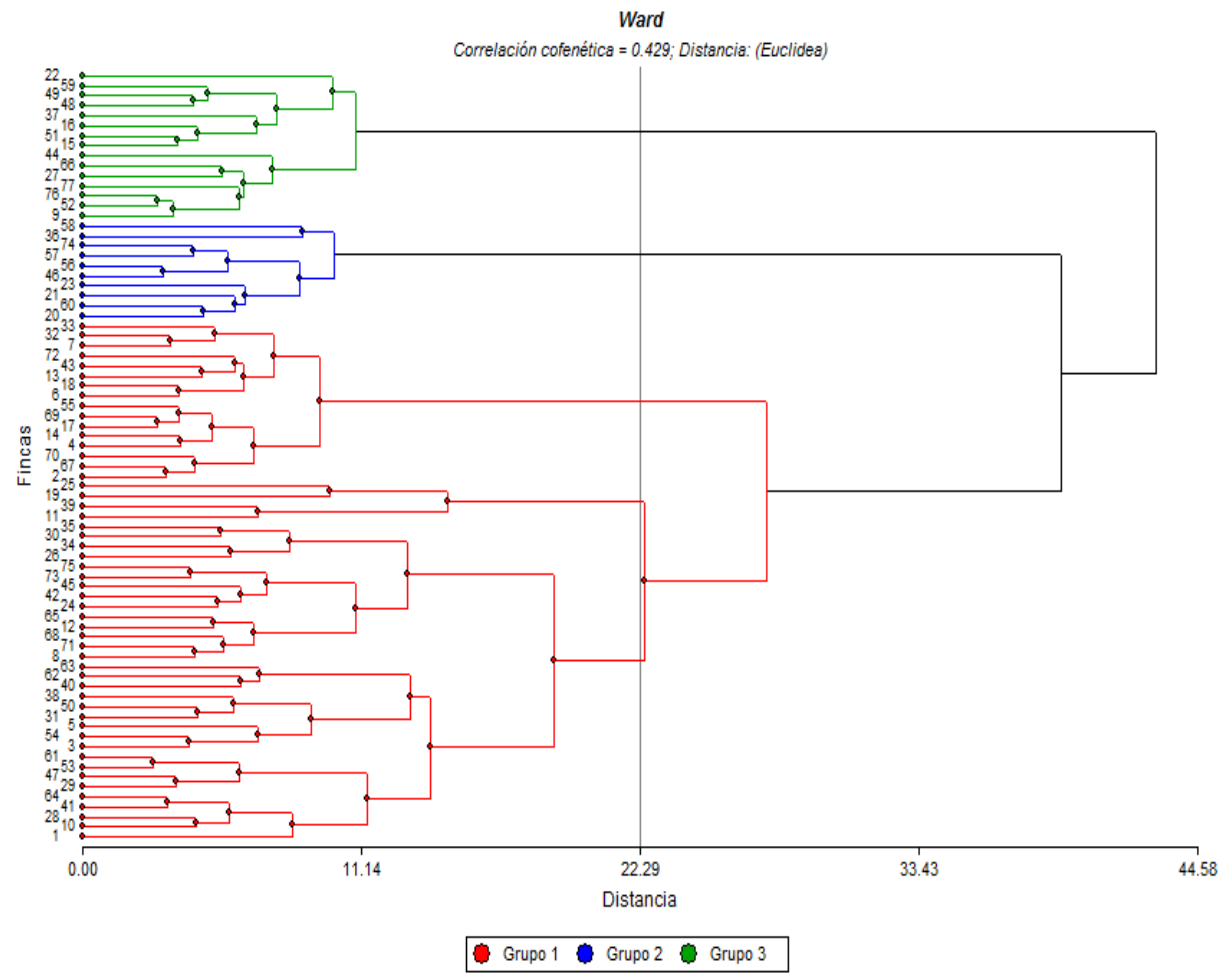

Figura 2. Agrupamiento de las fincas cacaoteras en Huicungo (San Martín).

incrementarse según la posición fisiográfica, pendiente y material parental del suelo (Lagos et al., 1994).

En el caso de variables del componente actividad agrícola, estas no mostraron diferencias significativas (Tabla 2). Pero, en las fincas del grupo I, las actividades productivas son más diversas, ya que no solo se produce cacao sino también cacao + cultivos anuales, e incluso hacen cacao + cultivos anuales + ganadería; mientras que los productores del grupo II y III, trabajan solo cacao o cacao + cultivos anuales (Figura 3). En general, la mayor diversidad de actividades productivas en la finca, aumenta la sostenibilidad económica, convirtiéndoles en sistemas integrales de producción. Según Ferro \& Meneses (1996), la diversificación de actividades eleva la rentabilidad de los predios, haciendo trabajos que no aumenten los niveles de riesgo y que no se abandone totalmente la actividad cacaotera.

En cuanto a las variables del componente social, todas resultaron significativas (Tabla 2). Las fincas del grupo I y II, un $80 \%$ de ellas, son orientados por extensionistas de instituciones de desarrollo y por la Cooperativa de Productores, mientras que las fincas del grupo III solo el $10 \%$ es atendido por extensionistas. Las fincas del grupo I y II, en su mayoría (90\%) pertenecen a una organización, mientras que las fincas del grupo III no pertenecen a ninguna organización (Figura 3). No cabe duda que es importante la adhesión a una organización agraria como son las cooperativas, ya que proveen de fortaleza económica y han desempeñado un papel muy relevante en el entorno rural donde están inmersas (Ruiz et al., 2006). Las actividades de estas instituciones están ligadas al ámbito de la producción, el comercio, las finanzas y las prestaciones de servicios, todo ello con la finalidad de mejorar las condiciones de vida del agricultor (Gómez, 2004). Entre los servicios que brinda una organización agraria está la de extensión rural, esta es una labor muy importante que realizan los profesionales e instituciones en su conjunto, puesto que la extensión agrícola es un tipo de educación no formal, que tiene como intención promover el conocimiento, en este caso agrícola, para poder facilitar el desarrollo de los agricultores (McLeod, 2003).

Las variables del componente económico resultaron significativas, en el caso de la Producción anual de cacao, de las fincas de los grupos I (963.46 kg.ha-1) y III (933.20 kg.ha ${ }^{-1}$ ) fueron estadísticamente similares y mayores que las del grupo II (623.40 kg.ha ${ }^{-1}$ ), según se muestra en la Tabla 2. El bajo rendimiento para las fincas del grupo II, se explica porque cuenta con plantas híbridas improductivas, realiza solamente dos deshierbos al año y no hace las labores de poda, siendo éstas dos últimas actividades 
Tabla 2. Comparación entre los tres grupos para todas las variables estudiadas.

\begin{tabular}{|c|c|c|c|c|c|c|c|}
\hline \multirow{2}{*}{$\mathrm{N}^{\circ}$} & & \multirow[b]{2}{*}{ VARIABLES } & \multicolumn{2}{|l|}{ p-valor } & \multicolumn{3}{|c|}{ GRUPOS } \\
\hline & & & Prueba F & $\begin{array}{l}\text { Chi- } \\
\text { cuadrado }\end{array}$ & $\begin{array}{l}\mathrm{I} \\
(\mathrm{n}=52)\end{array}$ & $\begin{array}{l}\text { II } \\
(n=10)\end{array}$ & $\begin{array}{l}\text { III } \\
(n=15)\end{array}$ \\
\hline \multicolumn{8}{|c|}{ FAMILIAR } \\
\hline 1 & $\mathrm{~F} 1$ & Edad del productor (años) & 0.9178 & & $46.21 \quad \mathrm{a}$ & 44.4 a & $45.87 \mathrm{a}$ \\
\hline 2 & F2 & \multirow{2}{*}{$\begin{array}{l}\text { Número personas por familia } \\
\text { Originario de la localidad } \\
\text { (dicotómica) }\end{array}$} & 0.3235 & & $4.02 \mathrm{a}$ & $4.40 \mathrm{a}$ & $3.73 \mathrm{a}$ \\
\hline \multirow[t]{2}{*}{3} & F3 & & & 0.4932 & $63.46 \%$ & $50.00 \%$ & $73.33 \%$ \\
\hline & \multicolumn{7}{|c|}{ RECURSOS NATURALES } \\
\hline 4 & RN1 & \multicolumn{2}{|l|}{ Ojo de agua en la finca (dicotómica) } & 0.5342 & $23.08 \%$ & $40.00 \%$ & $26.67 \%$ \\
\hline 5 & RN2 & \multicolumn{2}{|l|}{$\begin{array}{l}\text { Río o quebrada en la finca } \\
\text { (dicotómica) }\end{array}$} & 0.7152 & $32.69 \%$ & $20.00 \%$ & $33.33 \%$ \\
\hline 6 & RN3 & \multicolumn{2}{|l|}{ Uso de agua de pozo (dicotómica) } & 0.2027 & $50.00 \%$ & $80.00 \%$ & $60.00 \%$ \\
\hline 7 & RN4 & \multicolumn{2}{|l|}{$\begin{array}{l}\text { Contaminación del agua } \\
\text { (dicotómica) }\end{array}$} & 0.3403 & $13.46 \%$ & $0.00 \%$ & $20.00 \%$ \\
\hline 8 & RN5 & \multicolumn{2}{|l|}{ Erosión en la finca (dicotómica) } & 0.4763 & $11.54 \%$ & $0.00 \%$ & $6.67 \%$ \\
\hline 9 & RN6 & Número de especies forestales & 0.0101 & & $4.62 \mathrm{a}$ & $2.50 \mathrm{~b}$ & $4.00 \mathrm{a}$ \\
\hline 10 & RN7 & Área de bosque (ha) & 0.4583 & & $12.57 \mathrm{a}$ & $8.41 \mathrm{a}$ & 7.25 a \\
\hline \multirow[t]{2}{*}{11} & RN8 & Área de purma (ha) & 0.3526 & & 0.52 a & 0.20 a & $1.07 \mathrm{a}$ \\
\hline & ACTIVIDAD & AGRÍCOLA & & & & & \\
\hline \multirow[t]{5}{*}{12} & AA1 & Principales actividades (escala) & & 0.7543 & & & \\
\hline & & Solo cacao & & & $23.08 \%$ & $40.00 \%$ & $20.00 \%$ \\
\hline & & Cacao y cultivos anuales & & & $69.23 \%$ & $60.00 \%$ & $80.00 \%$ \\
\hline & & Cacao y ganadería & & & $1.92 \%$ & $0.00 \%$ & $0.00 \%$ \\
\hline & & Cacao, cultivos anuales y ganadería & & & $5.77 \%$ & $0.00 \%$ & $0.00 \%$ \\
\hline 13 & AA2 & Área de cacao (ha) & 0.6089 & & $2.37 \mathrm{a}$ & $2.14 \mathrm{a}$ & $2.02 \mathrm{a}$ \\
\hline 14 & AA3 & Edad del cacaotal (años) & 0.2926 & & 6.40 a & $6.30 \mathrm{a}$ & 6.73 a \\
\hline 15 & AA4 & Área total del fundo (ha) & 0.3988 & & 16.85 a & 11.60 a & $11.07 \mathrm{a}$ \\
\hline 16 & $\begin{array}{l}\text { AA5 } \\
\text { ECONÓMIC }\end{array}$ & $\begin{array}{l}\text { Área de cultivos anuales (ha) } \\
\text { O }\end{array}$ & 0.5188 & & 0.93 a & 0.85 a & 0.73 a \\
\hline 17 & E1 & Producción anual cacao 2011 (kg/ha) & $<0.0001$ & & $\begin{array}{l}963.46 \\
a\end{array}$ & $623.40 \mathrm{~b}$ & $933.20 \mathrm{a}$ \\
\hline 18 & E2 & Mano de obra (escala) & & 0.0112 & & & \\
\hline & & Familiar & & & $28.85 \%$ & $60.00 \%$ & $33.33 \%$ \\
\hline & & Particular & & & $19.23 \%$ & $40.00 \%$ & $26.67 \%$ \\
\hline & & Ambos & & & $51.92 \%$ & $0.00 \%$ & $40.00 \%$ \\
\hline 19 & E3 & Margen Bruto (S/. x ha)* & $<0.0001$ & & $\begin{array}{l}4.362 .53 \\
\mathrm{a}\end{array}$ & $2.562 .77 \mathrm{~b}$ & $4.414 .6^{\mathrm{a}}$ \\
\hline 20 & E4 & Financiamiento externo (dicotómico) & & $<0.0001$ & $100.00 \%$ & $30.00 \%$ & $80.00 \%$ \\
\hline & TECNOLOG & IA Y GESTION & & & & & \\
\hline 21 & TYG1 & $\begin{array}{l}\text { Área de construcciones }\left(\mathrm{m}^{2}\right) \\
\text { Genotipos cacao (escala) }\end{array}$ & 0.354 & $<0.0001$ & 59.65 a & 58.70 a & 63.93 a \\
\hline 22 & TYG2 & Híbrido & & & $0.00 \%$ & $10.00 \%$ & $0.00 \%$ \\
\hline & & CCN51 & & & $90.38 \%$ & $10.00 \%$ & $100.00 \%$ \\
\hline & & CCN51+ICS-95 & & & $9.62 \%$ & $0.00 \%$ & $0.00 \%$ \\
\hline & & CCN51 + hibrido & & & $0.00 \%$ & $80.00 \%$ & $0.00 \%$ \\
\hline 23 & TYG3 & Realiza poda (dicotómico) & & $<0.0001$ & $100.00 \%$ & $0.00 \%$ & $100.0 \%$ \\
\hline 24 & TYG4 & Número de deshierbos al año & $<0.0001$ & & 3.92 a & $2.30 \mathrm{~b}$ & $4.00 \mathrm{a}$ \\
\hline 25 & TYG5 & $\begin{array}{l}\text { Control cultura de plagas } \\
\text { (dicotómico) }\end{array}$ & & 0.0421 & $73.08 \%$ & $100.00 \%$ & $86.67 \%$ \\
\hline 26 & TYG6 & Venta a la Cooperativa (dicotómico) & & $<0.0001$ & $96.15 \%$ & $90.00 \%$ & $6.67 \%$ \\
\hline 27 & $\begin{array}{l}\text { TYG7 } \\
\text { SOCIAL }\end{array}$ & Lleva registros (dicotómico) & & $<0.0001$ & $96.15 \%$ & $90.00 \%$ & $0.00 \%$ \\
\hline 28 & S1 & $\begin{array}{l}\text { Aconsejado por extensionista } \\
\text { (dicotómico) }\end{array}$ & & $<0.0001$ & $88.46 \%$ & $80.00 \%$ & $13.33 \%$ \\
\hline 29 & S2 & $\begin{array}{l}\text { Pertenece } \\
\text { (dicotómico) }\end{array} \quad$ organización & & $<0.0001$ & $96.15 \%$ & $90.00 \%$ & $0.00 \%$ \\
\hline 30 & S3 & Tiempo organizado (años) & $<0.0001$ & & $4.17 \mathrm{a}$ & $3.80 \mathrm{a}$ & $0.00 \mathrm{~b}$ \\
\hline
\end{tabular}

muy importantes para incrementar la producción en el resultados para el Margen Bruto (Tabla 2), mostraron cultivo de cacao (Arévalo \& Zúñiga, 2002). Los las mismas tendencias que en el caso anterior, los 
valores de los grupos I (S/. 4362.53) y III (S/. 4414.60) fueron estadísticamente similares y mayores que las del grupo II (S/. 2562.77). Las fincas del grupo II trabajan en gran medida con la mano de obra familiar (60\%), mientras que en las fincas de los grupos I (28\%) y III (33\%), la disponibilidad de mano de obra familiar es menor; pero que complementan con la contratación de mano de obra particular (Tabla 2), al parecer adecuadamente seleccionada y supervisada, que permitiría la mejora de la producción. Esta situación favorece a los programas de extensión y asistencia técnica (Sánchez, 1990), porque el personal especializado tendrá mayor tiempo y experiencia para implementar las recomendaciones técnicas para este cultivo.

Respecto a la capacidad de recibir financiamiento externo, solo la tienen en mayor medida las fincas I y III, mientras que las fincas del grupo II solo un 30 por ciento de ellas disponen de financiamiento externo (Tabla 2), los productores del grupo I el financiamiento externo es invertido en sus fincas para destinar a las labores de poda donde el productor tomo conciencia que debe de invertir en ésta labor para poder fortalecer al cultivo e incrementar la producción. Este bajo porcentaje de acceso al crédito se deba tal vez a que en muchas casos el pequeño agricultor no tiene dominio pleno del terreno o no dispone de otros bienes que le permitan respaldar el crédito (Sánchez, 1990). Los estratos sociales rurales con un mayor acceso a todos los tipos de activos, tienen una mayor posibilidad de innovar, accede a las nuevas oportunidades provocadas por el cambio local, nacional e internacional, como, por ejemplo, nuevos mercados para cultivos de alto valor; establecer vínculos más sólidos entre la producción primaria, la industria y los servicios (Berdegué, 2005).

Con respecto a las variables del componente tecnología y gestión (Tabla 2), las fincas del grupo III solo tienen plantas de CCN51, mientras que las fincas del grupo I tienen plantas policlonales como son CCN51 + ICS95 y las fincas del grupo II, tienen plantaciones híbridas asociadas con CCN-51. Diversos autores señalan que las plantaciones policlonales brindan muchas ventajas, comparadas con las monoclonales, como tolerancia a las enfermedades y a los veranos prolongados, además favorece la polinización (Arévalo \& Zuñiga, 2004). Además en este caso, el grupo II, tiene la desventaja de tener plantas hibridas improductivas, como consecuencia de la segregación que presenta una población propagada a partir de semilla botánica y que conlleva a un bajo rendimiento (Arévalo \& Zúñiga, 2002).

Respecto al manejo de podas, el $100 \%$ de las fincas de los grupos I y III realizan las labores de poda en sus plantaciones; mientras que las del grupo II no realizan esta labor (Tabla 2). Según López (1991), la poda es una práctica cultural muy importante porque de ella depende, en buena parte, la formación del árbol
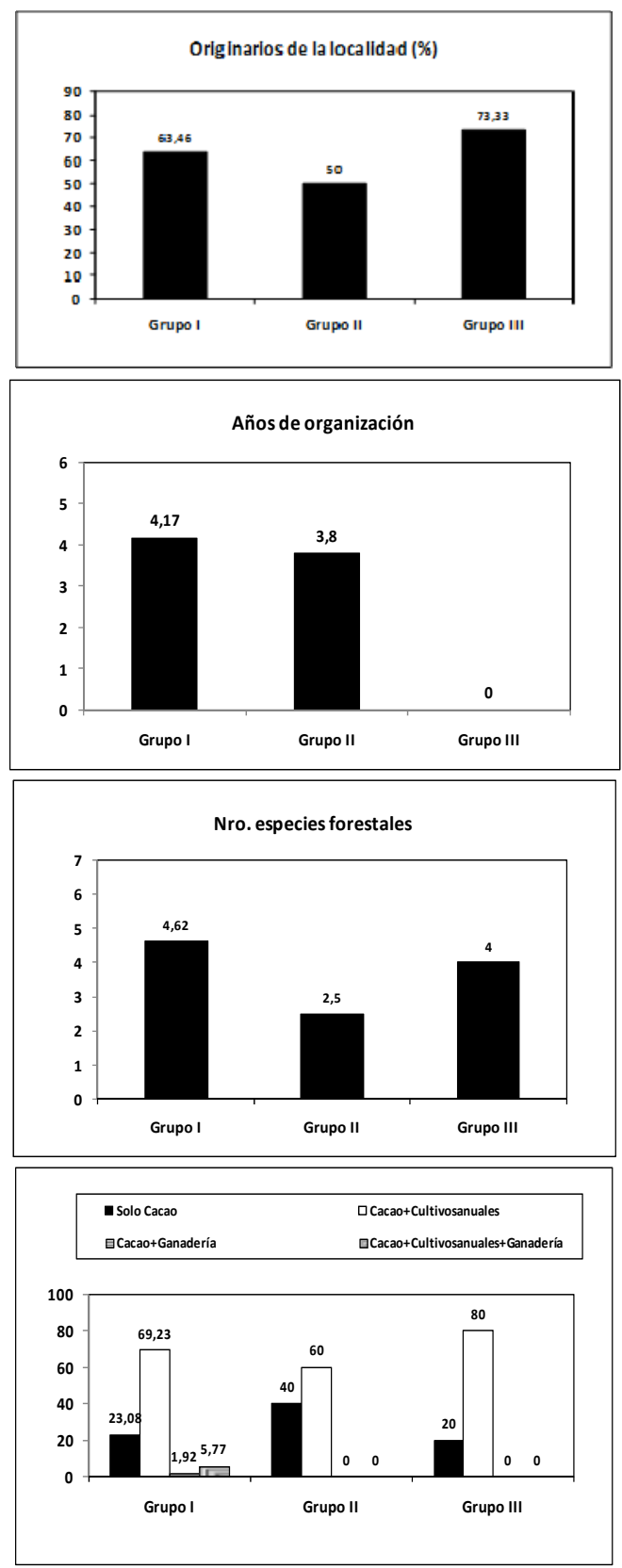

Figura 3. Procedencia de los productores, número de especies forestales en parcela, años de pertenecer a organización de productores y actividades realizadas en los tres grupos de fincas con cacao encontradas en la zona de estudio.

y a su vez una buena producción. La poda se debe realizar teniendo en cuenta los criterios fisiológicos que permitan un buen comportamiento del árbol y una alta productividad. Para una mejor actividad fotosintética, debe haber una buena captación de luz solar, de manera práctica esto se obtiene despuntando algunas ramas superiores para aumentar la entrada de 
luz a las ramas inferiores y por consiguiente la exposición de las hojas a la luz solar (Arévalo \& Zúñiga, 2002).

En cuanto al control cultural de plagas (Tabla 2), el grupo II en su totalidad lo realiza manualmente, sin embargo el grupo I, para realizar el control de plagas utilizan control químico en un $26.92 \%$ y el grupo III en un $13 \%$. El control químico se hace aplicando óxido cuproso al $75 \%$ para proteger a las flores y frutos en los primeros cuatro meses, estado crítico para el ataque de los hongos (Arévalo \& Zúñiga, 2004) y es que un solo hongo como Phytophtora sp. puede reducir la producción de un 10 al 20\% (Porras \& Sánchez, 1991). La forma tradicional de controlar plagas y enfermedades en diversos cultivos, entre ellos el cacao, ha sido usando plaguicidas; pero esta práctica trajo problemas de contaminación ambiental que han impactado negativamente en la biodiversidad de los ecosistemas, en la seguridad y salud pública, lo que ha conducido a la búsqueda y desarrollo de alternativas ecológicas (Zavaleta, 1999).

En relación a las malezas, en general se acepta que estas son inútiles e indeseables; su crecimiento es agresivo, vigoroso y competitivo (Labrada \& Parker, 1994), lo que hace que esta planta tenga un impacto negativo en las fincas cacaoteras. Al evaluar el número de deshierbo por año, los valores obtenidos en las fincas de los grupos I y III fueron estadísticamente similares y mayores que las fincas del grupo II, donde se hace solamente 2.30 deshierbos/año (Tabla 2). La falta de control de malezas en las fincas del grupo II, permite la competencia por nutrientes y agua entre estas especies no deseables y el cultivo de cacao. Además, un campo libre de malezas, facilita la ejecución de otras actividades propias del cultivo (Arévalo \& Zúñiga, 2004).

En cuanto a la tecnología y gestión de las fincas, la Tabla 2, muestra que las fincas del grupo III no llevan registros de producción, mientras que la mayoría (90\%) del grupo I y II si los llevan. Además, la venta del cacao a la Cooperativa asegura el fortalecimiento de estas organizaciones, por ello el $90 \%$ de las fincas de los grupos I y II realizan la venta a sus cooperativas, mientras que en las fincas del grupo III, solamente el $6.67 \%$ lo hace a través de las cooperativas, la mayor parte vende a los intermediarios.

Por otra parte, en la Figura 4, se muestra que el primer componente (CP1) explica un 65.8\% de la variabilidad de la formación de los grupos y separa las variables Tecnología y Gestión, Económico, Familiar y Actividad Agrícola del resto de variables, por tanto la mayor variabilidad entre los grupos se explica con estas variables. El Grupo I está más asociado a las variables Actividad Agrícola, mientras que el Grupo II, está más asociado a la variable Económica; por otro lado, el Grupo III se encuentra asociado a la variable

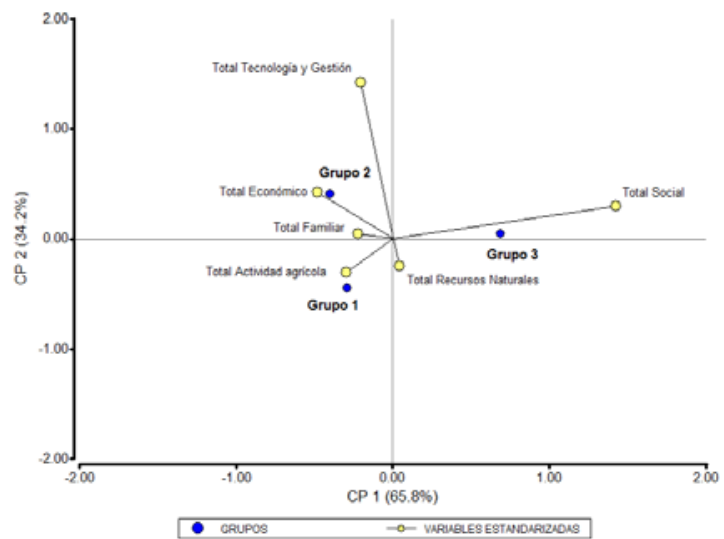

Figura 4. Biplot para las variables de clasificación que muestra la variabilidad de los grupos formados.

Social. El segundo componente (CP2) separa las variables Tecnología y Gestión, Económico, Social y Familiar de las variables Actividad Agrícola y Recursos Naturales.

En función de todas las variables utilizadas, las fincas quedaron suficientemente discriminadas en tres grupos distintos entre sí. El análisis discriminante explica el 62.24 por ciento de la variabilidad en el primer eje canónico, sin cometer error de clasificación cruzada. Sin embargo, debido al reducido número de fincas del Grupo II se formó una gran elipse de predicción alrededor de las fincas, indicando una mayor variabilidad entre las mismas fincas (Figura 5).

Las variables identificadas de mayor poder discriminante entre todas las variables evaluadas fueron Área de bosque, Área total del fundo, Área de cultivos anuales, Margen bruto por hectárea, Número de deshierbo por año, Lleva registros y Pertenece a una organización. Las variables de mayor poder discriminante fueron el Margen bruto (S/. x ha), que considera los ingresos por venta de la producción de grano y los costos de producción, el cual es

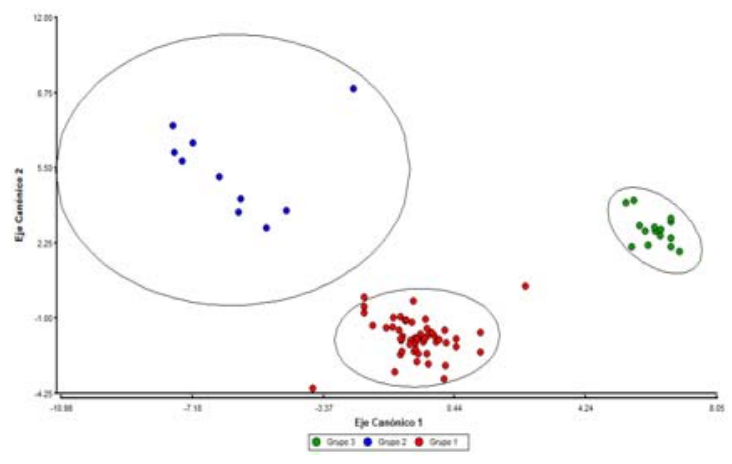

Figura 5. Representación de la distribución de las fincas por grupos de acuerdo a todas las variables utilizadas en el estudio. 
responsable de la separación de las fincas del Grupo I del resto de grupos en el primer eje canónico; asimismo el uso de registros y si pertenece a alguna organización permiten esta diferenciación.

Se concluyó que las fincas cacaoteras están agrupadas en tres tipos, significativamente diferenciados entre sí. El grupo I fue el mayoritario (68\% de las fincas), se caracteriza por desarrollar otras actividades paralelas al cultivo del cacao, tiene un sistema de producción convencional, con plantaciones policlonales y un rendimiento promedio de 963.46 kg.ha-1 ${ }^{-1}$ cifra que supera la media regional y nacional. El grupo II (13 \% de fincas) reúne básicamente fincas de producción orgánica, donde predominan plantaciones hibridas asociadas con CCN51 que tienen un rendimiento que no superan el rendimiento regional; pero si el nacional. En el grupo III, se encuentran plantaciones monoclonales usando únicamente el CCN-51. Los resultados sugieren que si en el futuro se implementan proyectos de mejora tecnológica u otros, estos deberían ser específicos para cada uno de los tres grupos encontrados en esta zona de estudio.

\section{Literatura citada.}

Altieri M. 2002. Agroecología: Bases científicas para una agricultura sustentable. Guaíba, BR. Agropecuaria.

Andrade M. 1985. Metodología para tipificar tecnologías de producción en fincas cafetaleras mediante las técnicas de análisis de componentes principales y clasificación automática jerárquica. Tesis de grado. Facultad de Agronomía. Universidad de Costa Rica. pp: 111.

Arévalo G. \& Zúñiga C. 2002. Manejo Integrado de Enfermedades del cacao. Banda de Shilcayo - San Martín: Instituto de Cultivos Tropicales (ICT).

Arévalo G. \& Zúñiga C. 2004. Manejo integrado del cultivo de cacao y transferencia de tecnología en la amazonía peruana. Tarapoto. San Martín. Instituto de Cultivos Tropicales (ICT).

Berdegué J. 2005.Sistemas de innovación favorables a los pobres. Backgroundpaper. IFAD.

Corven J. 1993. Asociación de cultivos con cacao, aspectos económicos. Seminario Regional "Sombras y cultivos asociados con cacao". CATIE. Costa Rica.

Escobar G. \& Berdegué J. 1990. Tipificación de sistemas de producción agrícola. RIMISP. Santiago de Chile.

Escobar G. 1986. Seminario: Clasificación de sistemas de fincas para generación y transferencia de tecnología apropiada. Panamá.

Ferro J. \& Meneses O. 1996. Cuadernos de Desarrollo Rural (37).

Gómez J. 2004. La Reforma de la PAC y la importancia de las cooperativas agrarias en la vertebración socioeconómica y territorial del medio rural. ERIA 63: 73-90.

Jiménez F. 2002. Metodología rápida para estimar el manejo de una microcuenca. En: Material didáctico del curso Manejo Integrado de cuencas Hidrográficas II. Turrialba, C.R. CATIE.

Labrada R. \& Parker C. 1994. Weed control in the context of integrated management. In: Weed Management for Developing Countries. FAO. Roma.

Lagos M., Espinoza J. \& Ortiz R. 1994. Erosión de los suelos en Chile. En: Erosión del suelo en América Latina. Suelos y Agua. FAO. Chile.

Lopez A. 1991. Diagnóstico de la situación del cacao en Guatemala. IICA. Guatemala.

McLeod W. 2003. Agricultural Extension, Rural Development and teh Food Security Challenge. FAO. Italy.

MINAG. 2005. Plan Estratégico, Cadena Agroproductiva del Cacao. IICA- CICAD- GTZ. Agosto. Lima.

Nair P. 1982. Soil productivity aspects of agroforestery. Nairobi: ICRAF.

Porras V. \& Sánchez J. 1991. Enfermedades del cacao. Tecnología, Comunicación y Desarrollo Nro1.

Raintree J. 1987. The state of the art in agroforestry diagnosis and design. Agroforestry Systems. 5(3): 219250.

Rodríguez W. 2002. Tipificación de los sistemas de fincas presentes en la micro cuenca del río Reventado. Revistas de las Sedes Regionales. 3(4): 53-62.

Rodríguez F. 2005. Las potencialidades y limitaciones del departamento de San Martín. Propuesta de Zonificación Ecológica y económica como base para el Ordenamiento Territorial.

Ruíz M., Hernández M. \& García E. 2006. Estado Actual de la Investigación sobre Sociedades Cooperativas en España. Revista de Economía Pública, Social y Cooperativa. 56: 65-86.

Sánchez J. 1990. Caracterización de la producción de cacao en Honduras. IICA-PROCACO-FHIA. Honduras.

Salazar R. 2012. Caracterización de sistemas agroecológicos para el establecimiento comercial de cacao (Theobroma cacao) en Talamanca. Tecnología en Marcha. 25(5).

Scheaffer R., Mendenhall W. \& Ott L. 1987. Elementos de muestreo. Editorial Iberoamericano. México DF.

Somarrriba E. 1998. Diagnóstico y Diseño Agroforestal. Agroforesteria en las Américas. 5(17-18): 68-72.

Somarriba E. 2006. Como analizar y mejorar la sombra en los cacaotales. CATIE, Bolivia. Taller Regional de Aplicación Tecnológica en el cultivo del cacao. ACCESO-IICA- USAID- CF- CICAD/OEA.

Vega M. \& Somarriba E. 2005. Planificación Agroforestal de fincas Cacaoteras orgánicas del Alto Beni, Bolivia. Agroforesteria en las Américas 43-44.

Zavaleta E. 1999. Alternativas de Manejo de la Enfermedades de las plantas. Terra 17(3).

\footnotetext{
${ }^{1}$ Biodiver Perú. Jr. Lamas 667. Tingo María. Huánuco. Perú.

2 Universidad Nacional Agraria La Molina. Facultad Agronomía. Dpto. Fitotecnia. La Molina. Lima. Perú. Correo electrónico: ajo@lamolina.edu.pe

${ }^{3}$ Universidad Estatal Península de Santa Elena. Av. Principal La Libertad. Santa Elena. Ecuador.
} 